\title{
On utility-based derivative pricing with and without intermediate trades
}

\author{
Jan Kallsen* and Christoph Kühn ${ }^{\dagger}$
}

\begin{abstract}
The neutral valuation approach for contingent claims in incomplete markets is based on the assumption that investors are identical utility maximizers and that derivative supply and demand are balanced. It is closely related to (marginal) utility-based pricing in the sense of Hugonnier et al. (2005), where however only buy-and-hold investments in the derivative are possible.

This paper contains four results: Firstly, it is shown that neutral derivative prices exist in discrete-time markets with finite time horizon. They are characterized as martingales relative to certain finitely additive set functions. Secondly, it may happen that not any static utility-based derivative price can be extended to yield a neutral price process. Thirdly, neutral derivative prices may not exist in continuous-time markets. Finally, we consider the situation of finite utility on the whole real line.
\end{abstract}

Key words: Neutral derivative pricing, utility-based pricing, incomplete markets, martingales under finitely additive set functions

JEL Classification: G11, G13, D52

Mathematics Subject Classification (2000): 91B24, 60G48, 91B16, 46E99

\section{Introduction}

This paper concerns the derivative pricing problem in incomplete markets. More specifically, suppose that the price processes of a number of underlyings are exogenously given. We are interested in the initial price or even the whole price process of one or several contingent claims, which are given in terms of their terminal payoff at maturity. Unless we are in the fortunate situation of a complete market model, arbitrage arguments typically do not

\footnotetext{
*HVB-Stiftungsinstitut für Finanzmathematik, Zentrum Mathematik, TU München, D-85747 Garching bei München, Germany, (e-mail: kallsen@ma.tum.de)

${ }^{\dagger}$ Frankfurt MathFinance Institute, Johann Wolfgang Goethe-Universität, D-60054 Frankfurt am Main, Germany, (e-mail: ckuehn@math.uni-frankfurt.de)
} 
suffice to fix the prices resp. price processes of these options uniquely. Stronger assumptions have to be made if one wants to pursue this goal.

Relying on classical principles in economic theory, utility-based approaches have received a lot of attention in recent years. They all involve assumptions on the preferences of market participants, which are expressed in terms of utility functions. However, they differ considerably in their economic viewpoint and hence in their precise axiomatic framework.

Utility-based indifference pricing (cf. e.g. $[12,6]$ ) takes the perspective of a particular counterparty and fixes the number of shares of the claim (say, $\delta=1$ for an option buyer or $\delta=-1$ for an option seller). The indifference premium or reservation price is a price such that the optimal expected utility among all portfolios containing the prespecified number of options coincides with the optimal expected utility among all portfolios without options. Put differently, the investor is indifferent to including the option into the portfolio. Note that utility indifference pricing is an asymmetric, static, size-dependent concept, i.e. it distinguishes between buyer and seller, does not allow for intermediate trades of the contingent claim, and depends on the number of options $\delta$.

[5] and [18] consider the limit of utility indifference prices if $\delta$ tends to 0 . More precisely, they propose to call an initial option price fair if the investor is marginally indifferent versus including the option in the portfolio or not. One may call these prices marginal utility indifference prices.

The valuation concepts discussed in $[9,16,17,13]$ are rather based on symmetric, sizeindependent axioms, which carry a partial equilibrium flavour. Assume that a certain derivative is introduced in zero-net supply into the market. Since any option that is bought has to be sold by another investor, this leads to a clearing condition for the derivatives market. We suppose that all investors in this secondary market are identical utility maximizers with a given profile. If they behave optimally, the option prices must be such that the utilityoptimal portfolio of the average investor contains no contingent claim. Such prices are called neutral in [16, 17] and marginal utility-based prices in [13]. Recall that the prices of the underlyings are assumed to be exogenously given and that they are not affected by derivative investors. Therefore these concepts differ somewhat from general equilibrium theory applied to the market as a whole.

Neutral prices of $[16,17]$ and marginal utility-based prices in the sense of [13] differ as far as investment opportunities in the derivative are concerned. [13] consider only buyand-hold strategies in the claim, whereas intermediate trades are allowed in $[16,17]$ (cf. also $[10,15]$ as earlier references). Nevertheless, the main idea is the same: The option is assumed to be traded at a price such that its presence does not lead to additional utility.

By way of duality, portfolio optimization problems are linked to some dual minimization problems in the set of equivalent martingale measures or some related set of stochastic processes which generalizes the class of density processes of equivalent martingale measures. These measures can be interpreted as pricing rules for contingent claims. It has been observed that the marginal and neutral derivative prices of $[5,18,16,17,13]$ are obtained as expectations under the dual minimizer if the latter is a true equivalent martingale measure. 
This measure is called neutral pricing measure in $[16,17]$. In general, however, the dual minimizer in the sense of [19] need not be a martingale, which means that the neutral pricing measure fails to exist. In the language of [3], the dual minimization problem is solved by some non-unique finitely additive set function. [13] investigate the existence and uniqueness of marginal utility-based prices in this general case. It turns out that the non-uniqueness of the dual minimizer always leads to non-unique utility-based prices for some claims.

In this paper a similar analysis is undertaken for neutral derivative pricing in the dynamic sense where contingent claims can be traded at any time. Since the static concept of [13] and the dynamic version considered here coincide in one-period models, the results of [13] show that uniqueness of neutral price processes does not hold in general either.

In Section 2 we show that neutral derivative prices exist in multiperiod discrete-time markets. They are characterized as martingales relative to some finitely additive set function which solves the dual minimization problem in [3].

However, it may happen that not all dual minimizers in [3] can be used as a pricing rule for derivatives. In this case, the set of marginal utility-based prices in the sense of [13] may be strictly larger than the corresponding set of initial neutral prices. This leads to a phenomenon which seems paradoxical on first glance: If the claim is traded at a certain price at time 0 only, then the utility maximizers do not profit from its presence. But if a market for the claim exists also between time 0 and maturity $T$, trading the claim will lead to additional utility - no matter how the price process strictly between 0 and $T$ is chosen (cf. Example 3.3).

Moreover, we show that in continuous time even the existence of neutral prices may break down: In an arbitrage-free market with finite expected utility, there may be no way to price a given bounded claim such that the maximal utility is not raised in the extended market where dynamic trading of the claim is possible (cf. Example 3.4).

The situation is different for utility functions that are finite on the whole real line. In this case the dual minimizer is always a true probability measure which is absolutely continuous with respect to the original measure (cf. [2] and references therein). This leads to essentially unique neutral price processes. To be more precise, some non-uniqueness may still occur on the set where the density process of the dual minimizer vanishes. Moreover, neutral price processes may fail to exist if the dual minimizer is strictly absolutely continuous. This is discussed in Section 4.

We use the dot notation for stochastic integrals. If $X$ is a semimartingale, $L(X)$ denotes the set of $X$-integrable predictable processes in the sense of [14], III.6.17. Superscripts refer generally to coordinates of a vector or vector-valued process rather than powers.

\section{Existence of neutral price processes}

Before we turn to the existence of neutral derivative price processes in discrete time, let us introduce a general setup which makes sense in continuous time as well. Fix a terminal date $T \in \mathbb{R}_{+}$and a filtered probability space $\left(\Omega, \mathscr{F},\left(\mathscr{F}_{t}\right)_{t \in[0, T]}, P\right)$. We distinguish two kinds 
of securities in the markets, namely underlyings and derivatives, which can both be traded dynamically. The discounted price process $\left(S^{1}, \ldots, S^{m}\right)$ of the underlyings is assumed to be an $\mathbb{R}^{m}$-valued semimartingale which is given beforehand.

Moreover, let bounded $\mathscr{F}_{T}$-measurable random variables $H^{1}, \ldots, H^{n}$ represent the discounted terminal payoff of $n$ contingent claims. Their price process has yet to be determined. At this stage, we call any bounded $\mathbb{R}^{n}$-valued semimartingale $\left(S^{m+1}, \ldots, S^{m+n}\right)$ derivatives price process if $S_{T}^{m+i}=H^{i}$ for $i=1, \ldots, n$.

Altogether, $S=\left(S^{1}, \ldots, S^{m+n}\right)$ is the discounted price process of the $m+n$ securities in the market. Trading strategies $\psi \in L(S)$ are called admissible if their discounted value process is bounded from below, i.e. there exists some $c \in \mathbb{R}$ such that $\psi \cdot S \geq c$ outside some $P$-null set.

We assume that investors are identical utility maximizers with initial endowment $v \in$ $(0, \infty)$. Their preferences are modelled by a strictly concave utility function $u: \mathbb{R} \rightarrow$ $\mathbb{R} \cup\{-\infty\}$ which is continuously differentiable on $(0, \infty)$ and satisfies $u(x)=-\infty$ for $x<0, \lim _{x \rightarrow 0} u^{\prime}(x)=\infty, \lim _{x \rightarrow \infty} u^{\prime}(x)=0$, and $\limsup _{x \rightarrow \infty} \frac{x u^{\prime}(x)}{u(x)}<1$ (i.e. it has asymptotic elasticity strictly less than 1 in the sense of [19], Definition 2.2).

An admissible trading strategy $\varphi$ is called optimal if it maximizes the expected utility of terminal wealth $\psi \mapsto E\left(u\left(v+\psi \cdot S_{T}\right)\right)$ over all admissible strategies $\psi \in L(S)$. Here, we set $E\left(u\left(v+\psi \cdot S_{T}\right)\right):=-\infty$ if $E\left(-u\left(v+\psi \cdot S_{T}\right) \vee 0\right)=\infty$. We assume that the maximal expected utility which is achievable by trading in the underlyings is finite and attained by some strategy, i.e.

$$
\begin{aligned}
& \sup \left\{E\left(u\left(v+\psi \cdot S_{T}\right)\right): \psi \text { admissible with } \psi^{i}=0 \text { for } i=m+1, \ldots, m+n\right\} \\
& =E\left(u\left(v+\varphi \cdot S_{T}\right)\right)<\infty
\end{aligned}
$$

for some admissible $\varphi$ with $\varphi^{i}=0$ for $i=m+1, \ldots, m+n$.

Proposition 2.1 Under condition (2.1) the semimartingale $\left(S^{1}, \ldots, S^{m}\right)$ satisfies the condition of no free lunch with vanishing risk (NFLVR) in the sense of [7].

Proof. Assume that NFLVR does not hold. Then there exist some $f \in L_{+}^{0}(\Omega, \mathscr{F}, P) \backslash\{0\}$ and some sequence of admissible strategies $\psi^{(n)}, n \in \mathbb{N}$ which trade only with $S^{1}, \ldots, S^{m}$ and satisfy $f \leq \frac{1}{n}+\psi^{(n)} \cdot S_{T}$. Let $\varphi$ be an optimal strategy for $S^{1}, \ldots, S^{m}$ in the sense of (2.1) and set $\varphi^{(n)}:=\left(1-\frac{2}{v n}\right) \varphi+\psi^{(n)}$. For $n \geq 2 / v$ the strategies $\varphi^{(n)}$ are admissible. Moreover, $v+\varphi^{(n)} \cdot S_{T} \geq\left(v+\varphi \cdot S_{T}\right) \wedge \frac{v}{2}$, which implies that the sequence $u\left(v+\varphi^{(n)} \cdot S_{T}\right)$ is bounded from below by an integrable random variable. Since $\liminf _{n \rightarrow \infty} u\left(v+\varphi^{(n)} \cdot S_{T}\right) \geq u\left(v+\varphi \cdot S_{T}+f\right)$ almost surely, Fatou's lemma and the monotonicity of $u$ yield

$$
\liminf _{n \rightarrow \infty} E\left(u\left(v+\varphi^{(n)} \cdot S_{T}\right)\right) \geq E\left(u\left(v+\varphi \cdot S_{T}+f\right)\right)>E\left(u\left(v+\varphi \cdot S_{T}\right)\right),
$$

which contradicts the optimality of $\varphi$ in (2.1). Thus NFLVR holds.

If contingent claims are in zero net supply and if their market clears, we end up with neutral prices in the following sense: 
Definition 2.2 We call derivative price processes $S^{m+1}, \ldots, S^{m+n}$ neutral price processes if there exists an admissible strategy $\varphi$ which is optimal for $S=\left(S^{1}, \ldots, S^{m+n}\right)$ and satisfies $\varphi^{m+1}=\ldots=\varphi^{m+n}=0$.

In finite probability spaces neutral derivative prices are obtained as conditional expectations under some specific equivalent martingale measure (cf. [16]). Often - but not always this extends to continuous-time semimartingale markets. This neutral pricing measure plays a key role in the dual approach to the portfolio optimization problem in the primary market. In order to obtain existence and duality results in a general semimartingale model, [19] and [3] go beyond the set of equivalent martingale measures. We follow here [3] by considering the dual space $\left(L^{\infty}\right)_{+}^{*}$, which can be identified with the set of nonnegative finitely additive set functions (cf. [8], Theorem IV.8.16).

For $Q \in\left(L^{\infty}\right)_{+}^{*}$ the operator $\langle Q, \cdot\rangle$ can be extended to any random variable which is bounded from below, namely by setting $\langle Q, X\rangle:=\lim _{n \rightarrow \infty}\langle Q, X \wedge n\rangle \in(-\infty, \infty]$. If $\langle Q,|X|\rangle\left\langle\infty\right.$, we set $\langle Q, X\rangle:=\left\langle Q, X^{+}\right\rangle-\left\langle Q, X^{-}\right\rangle$for arbitrary random variables $X$. Moreover, recall that any $Q \in\left(L^{\infty}\right)_{+}^{*}$ can be uniquely written as $Q=Q^{r}+Q^{s}$ with some measure $Q^{r}$ (the regular part) and some purely finitely additive set function $Q^{s}$ (the singular part, cf. [8], Definition III.7.7 and Theorem III.7.8 for this Yosida-Hewitt decomposition). $Q^{r}$ is absolutely continuous with respect to $P$.

In Theorem 2.5 it is shown that neutral price processes are martingales relative to neutral set functions, which are defined as follows:

Definition 2.3 In this definition we exceptionally consider the market without contingent claims, i.e. $S=\left(S^{1}, \ldots, S^{m}\right)$ denotes the price process of the underlyings and $\varphi, \psi$ below are $\mathbb{R}^{m}$-valued processes.

Let $Q \in\left(L^{\infty}\right)_{+}^{*}$ with $\langle Q, 1\rangle=1$ and denote by $Q=Q^{r}+Q^{s}$ its Yosida-Hewitt decomposition. We call $Q$ neutral set function if there exists some admissible strategy $\varphi$ and some $y \in(0, \infty)$ such that

1.

$$
y \frac{d Q^{r}}{d P}=u^{\prime}\left(v+\varphi \cdot S_{T}\right)
$$

2. $\left\langle Q, \psi \cdot S_{T}\right\rangle \leq 0$ for all admissible strategies $\psi$,

3. $\left\langle Q, \varphi \cdot S_{T}\right\rangle=0$,

4. $\left\langle Q^{s}, v+\varphi \cdot S_{T}\right\rangle=0$.

We denote by $\mathscr{Q}$ the set of neutral set functions. It is nonempty by [3].

$\mathscr{Q}$ is the set of dual minimizers in the extended dual domain from [3]. Note that the regular parts of the elements of $\mathscr{Q}$ coincide. If $Q$ is a neutral set function, then the corresponding $\varphi$ is an optimal strategy for $\left(S^{1}, \ldots, S^{m}\right)$.

It is natural to define $Q$-martingales in the following way: 
Definition 2.4 Let $Q$ be a nonnegative finitely additive set function with $\langle Q, 1\rangle=1$. An adapted process $X$ is called $Q$-martingale if $\left\langle Q,\left|X_{t}\right|\right\rangle<\infty$ and

$$
\left\langle Q, X_{t} 1_{A}\right\rangle=\left\langle Q, X_{T} 1_{A}\right\rangle
$$

for any $t \in[0, T]$ and any $A \in \mathscr{F}_{t}$. Multivariate martingales are defined componentwise as usual.

In contrast to probability measures, there may not exist a $Q$-martingale $X$ with terminal value $H$ for any given bounded random variable $H$ (cf. Example 3.3). If $Q$ is a neutral set function and hence $Q^{r} \sim P$, then $X$ is unique (up to a modification) if it exists at all. In finite discrete time the recursive procedure

$$
X_{T}:=H, \quad X_{t-1}:=E_{Q}\left(X_{t} \mid \mathscr{F}_{t-1}\right)
$$

yields such a $Q$-martingale if $Q$ is a probability measure. Conditional expectations may not exist for finitely additive set functions. Nevertheless, a similar construction is crucial for the proof of Theorem 2.5 although it does not necessarily lead to a $Q$-martingale.

From now on we work in a discrete-time market, i.e. the time domain is $\{0,1, \ldots, T\}$ rather than $[0, T]$. Our first main result treats existence of neutral derivative price processes and shows that they are recovered as martingales under neutral set functions.

Theorem 2.5 The set of neutral price processes $\left(S^{m+1}, \ldots, S^{m+n}\right)$ coincides with the set of $Q$-martingales having terminal value $\left(H^{1}, \ldots, H^{n}\right)$, where $Q$ runs through the set $\mathscr{Q}$ of neutral set functions. This set is nonempty, i.e. for at least one $Q \in \mathscr{Q}$ there exists a $Q$-martingale $\left(S^{m+1}, \ldots, S^{m+n}\right)$ with $S_{T}^{m+i}=H^{i}$ for $i=1, \ldots, n$.

PROOF. For ease of notation we assume $m=n=1$ in this proof and we write $H:=H^{1}$. The general case follows analogously.

Step 1: In the first two steps we show the existence of a neutral derivative price process. To this end, let $Q \in \mathscr{Q}$ with corresponding admissible strategy $\varphi$ for $S^{1}$ and $y>0$. We mimic the recursive definition (2.2) of a martingale for finitely additive set functions. We start with $S_{T}^{2}:=H$. Suppose that $S_{t}^{2}$ is already defined. On $\mathscr{F}_{t-1}$ define finitely additive set functions $Q_{t-1}, \mu_{t-1}^{+}, \mu_{t-1}^{-}, \mu_{t-1}$ by $Q_{t-1}:=\left.Q\right|_{\mathscr{F}_{t-1}}$ and

$$
\mu_{t-1}(A):=\mu_{t-1}^{+}(A)-\mu_{t-1}^{-}(A):=\left\langle Q,\left(S_{t}^{2}\right)^{+} 1_{A}\right\rangle-\left\langle Q,\left(S_{t}^{2}\right)^{-} 1_{A}\right\rangle .
$$

Denote by $Q_{t-1}=Q_{t-1}^{r}+Q_{t-1}^{s}$ etc. the Yosida-Hewitt decompositions of $Q_{t-1}, \mu_{t-1}^{+}, \mu_{t-1}^{-}$ into a regular and a singular part, respectively. Moreover, set $\mu_{t-1}^{r}:=\left(\mu_{t-1}^{+}\right)^{r}-\left(\mu_{t-1}^{-}\right)^{r}$ and $\mu_{t-1}^{s}:=\mu_{t-1}-\mu_{t-1}^{r}$. The regular parts are absolutely continuous to $P$. From $\mu_{t-1}^{ \pm} \leq$ $\left\|S_{t}^{2}\right\|_{\infty} Q_{t-1}$ it follows that $\left(\mu_{t-1}^{ \pm}\right)^{r} \leq\left\|S_{t}^{2}\right\|_{\infty} Q_{t-1}^{r}$ (using e.g. [3], Lemma A.1). Hence, we can define $S_{t-1}^{2}$ as the bounded Radon-Nikodym derivative of $\mu_{t-1}^{r}$ with respect to $Q_{t-1}^{r}$, i.e.

$$
S_{t-1}^{2}:=\frac{d \mu_{t-1}^{r}}{d Q_{t-1}^{r}}
$$


Step 2: We show that $S^{2}$ is a neutral price process. For any $t \in\{0, \ldots, T-1\}$ there exists an increasing sequence $\left(A_{k, t}\right)_{k \in \mathbb{N}}$ of $\mathscr{F}_{t}$-measurable sets with $P\left(A_{k, t}\right) \geq 1-1 / k$ and $Q_{t}^{s}\left(A_{k, t}\right)=0=\mu_{t}^{s}\left(A_{k, t}\right)$ (cf. [3], Lemma A.1). Thus we have

$$
\left\langle Q, \Delta S_{t}^{2} 1_{A_{k, t-1} \cap A}\right\rangle=\mu_{t-1}^{r}\left(A_{k, t-1} \cap A\right)-\left\langle Q_{t-1}^{r}, \frac{d \mu_{t-1}^{r}}{d Q_{t-1}^{r}} 1_{A_{k, t-1} \cap A}\right\rangle=0
$$

for $t=1, \ldots, T$ and $A \in \mathscr{F}_{t-1}$. Moreover, the sequence

$$
T_{k}(\omega):=\inf \left\{t \geq 0: \omega \notin A_{k, t}\right\} \wedge T, \quad k \in \mathbb{N},
$$

is localizing, i.e. $P\left(T_{k}=T\right) \rightarrow 1$ as $k \rightarrow \infty$.

For fixed $k \in \mathbb{N}$ consider the optimization problem

$$
\sup \left\{E\left(u\left(v+\psi^{1} \cdot S_{T}^{1}+\psi^{2} \cdot\left(S^{2}\right)_{T}^{T_{k}}\right)\right):\left(\psi^{1}, \psi^{2}\right) \in \Theta\right\}
$$

with

$$
\Theta:=\left\{\psi=\left(\psi^{1}, \psi^{2}\right): \psi^{2} \text { bounded, } \psi \in L(S) \text {, and } v+\psi^{1} \cdot S^{1}+\psi^{2} \cdot\left(S^{2}\right)^{T_{k}} \geq 0\right\}
$$

Let $\psi=\left(\psi^{1}, \psi^{2}\right) \in \Theta$. Since $\psi^{2} \cdot S^{2}$ is bounded, $\psi^{1} \cdot S^{1}$ is bounded from below and hence $\left\langle Q, \psi^{1} \cdot S_{T}^{1}\right\rangle \leq 0$. Equation (2.3) and the boundedness of $\psi^{2}$ and $S^{2}$ imply that $\left\langle Q, \psi^{2} \cdot S_{T_{k}}^{2}\right\rangle=0$ because $\psi^{2} \cdot S_{T_{k}}^{2}$ can be approximated uniformly by linear combinations of random variables of the form $\Delta S_{t}^{2} 1_{A_{k, t-1} \cap A}$. Therefore we have

$$
\left\langle Q, \psi^{1} \cdot S_{T}^{1}+\psi^{2} \cdot S_{T_{k}}^{2}\right\rangle \leq 0
$$

We obtain

$$
\begin{aligned}
& E\left(u\left(v+\psi^{1} \cdot S_{T}^{1}+\psi^{2} \cdot S_{T_{k}}^{2}\right)\right) \\
& \leq E\left(V\left(y \frac{d Q^{r}}{d P}\right)+y \frac{d Q^{r}}{d P}\left(v+\psi^{1} \cdot S_{T}^{1}+\psi^{2} \cdot S_{T_{k}}^{2}\right)\right) \\
& \leq E\left(V\left(y \frac{d Q^{r}}{d P}\right)\right)+y\left\langle Q, v+\psi^{1} \cdot S_{T}^{1}+\psi^{2} \cdot S_{T_{k}}^{2}\right\rangle \\
& \leq E\left(V\left(y \frac{d Q^{r}}{d P}\right)\right)+y v \\
& \quad=E\left(u\left(v+\varphi \cdot S_{T}^{1}\right)\right),
\end{aligned}
$$

where $V(z):=\sup _{x>0}(u(x)-x z)$ denotes the conjugate function of $u$ and the equality is stated in [3], (4.1). Therefore $(\varphi, 0)$ is optimal for the optimization problem (2.4).

Let $\psi=\left(\psi^{1}, \psi^{2}\right)$ be an admissible strategy for $S=\left(S^{1}, S^{2}\right)$ with $v+\psi \cdot S \geq 0$. Then $\left(1-\frac{1}{k}\right) 1_{\llbracket 0, T_{k} \wedge R_{k} \rrbracket} \psi \in \Theta$, where $R_{k}:=\inf \left\{t \in\{0, \ldots, T-1\}:\left|\psi_{t+1}^{2}\right| \geq k\right\} \wedge T$, and we have

$$
E\left(u\left(v+\left(\left(1-\frac{1}{k}\right) 1_{\llbracket 0, T_{k} \wedge R_{k} \rrbracket} \psi\right) \cdot\left(S^{1},\left(S^{2}\right)^{T_{k}}\right)_{T}\right)\right) \geq E\left(u\left(\frac{v}{k}\right)\right)>-\infty .
$$


By Lemma 2.2 in [17] the optimality of $(\varphi, 0)$ for problem (2.4) implies that

$$
E\left(u^{\prime}\left(v+\varphi \cdot S_{T}^{1}\right)\left(\left(1-\frac{1}{k}\right) 1_{\llbracket 0, T_{k} \wedge R_{k} \rrbracket} \psi-(\varphi, 0)\right) \cdot\left(S^{1},\left(S^{2}\right)^{T_{k}}\right)_{T}\right) \leq 0 .
$$

Note that $\Theta$ does not fit in the requirements of Lemma 2.2, but for the proof it is sufficient that $\Theta$ is convex. $\left(\left(1-\frac{1}{k}\right) 1_{\llbracket 0, T_{k} \wedge R_{k} \rrbracket} \psi\right) \cdot S_{T}$ converges pointwise to $\psi \cdot S_{T}$ for $k \rightarrow \infty$. By

$$
\left(\left(1-\frac{1}{k}\right) 1_{\llbracket 0, T_{k} \wedge R_{k} \rrbracket} \psi\right) \cdot S_{T} \geq-v
$$

and since $u^{\prime}\left(v+\varphi \cdot S_{T}^{1}\right)$ and $u^{\prime}\left(v+\varphi \cdot S_{T}^{1}\right)\left(v+\varphi \cdot S_{T}^{1}\right)$ are integrable, Fatou's lemma yields that

$$
E\left(u^{\prime}\left(v+\varphi \cdot S_{T}^{1}\right)(\psi-(\varphi, 0)) \cdot S_{T}\right) \leq 0
$$

Applying the converse implication of [17], Lemma 2.2, it follows that

$$
(\varphi, 0) \text { is optimal for } S=\left(S^{1}, S^{2}\right) \text { among all } \psi \in L(S) \text { with } v+\psi \cdot S \geq 0 .
$$

Let $\psi=\left(\psi^{1}, \psi^{2}\right)$ be an admissible strategy for $S=\left(S^{1}, S^{2}\right)$ such that $v+\psi \cdot S \geq 0$ does not hold. Then $E\left(u\left(v+\psi \cdot S_{T}\right)\right)=-\infty$ or there exists a one-period arbitrage. But the latter is impossible in view of (2.5).

Step 3: Let $S^{2}$ be a $Q$-martingale with terminal value $H$ for some $Q \in \mathscr{Q}$. We show that $S^{2}$ is a neutral derivative price process.

The martingale property implies that $\left\langle Q, \Delta S_{t}^{2} 1_{A}\right\rangle=0$ for $t=1, \ldots, T$ and any $A \in$ $\mathscr{F}_{t-1}$, which in turn yields that the left-hand side of (2.3) equals 0 . The assertion follows now as in Step 2.

Step 4: Conversely, let $S^{2}$ be a neutral derivative price process. We show that $S^{2}$ is a $Q$-martingale for some $Q \in \mathscr{Q}$.

Since $\left(S^{1}, S^{2}\right)$ is a market with finite maximal expected utility, the set $\widetilde{\mathscr{Q}}$ of neutral set functions corresponding to the extended market $\left(S^{1}, S^{2}\right)$ is nonempty by [3]. Since $S^{2}$ is bounded, strategies of the form $\left(\psi^{1}, \psi^{2}\right):=\left(0, \pm 1_{A \times\{t\}}\right)$ with some $t \in\{1, \ldots, T\}$ and some $A \in \mathscr{F}_{t-1}$ are admissible. Thus $\pm\left\langle Q, \Delta S_{t}^{2} 1_{A}\right\rangle=\left\langle Q, \psi \cdot S_{T}\right\rangle \leq 0$ and hence $S^{2}$ is a $Q$-martingale for any $Q \in \widetilde{\mathscr{Q}} \subset \mathscr{Q}$.

If $Q$ is a probability measure, then neutral prices are unique (cf. [17], Corollary 3.5). This does not hold in general: In a one-period market, neutral prices coincide with marginal utility-based prices put forward by [13]. Theorem 3.1 in the latter paper shows that uniqueness generally ceases to hold (cf. also Section 3 in this context).

\section{Counterexamples}

This section contains counterexamples which may seem surprising on first glance. The first concerns the relation between marginal utility-based prices in the sense of [13] and the initial values of neutral price processes. The second dashes the hopes to extend Theorem 2.5 to continuous time. Both constructions are based on the one-period Example 5.1' in [19], which we sketch briefly for the convenience of the reader: 
Example 3.1 The risky asset $S^{1}$ starts in $S_{0}^{1}=1$. In $t=1$ it assumes values $\left(x_{k}\right)_{k \in \mathbb{N}}$ with probabilities $\left(p_{k}\right)_{k \in \mathbb{N}}$, where e.g. $x_{0}:=2, p_{0}:=5 / 6, x_{k}:=2^{-k}, p_{k}:=4^{-k} / 2$ for $k \geq 1$. The agent is endowed with initial capital $v=1$ and strives to maximize her expected logarithmic utility from terminal wealth. The example is designed such that the optimal terminal wealth is $S_{1}^{1}$ (buy one risky asset) and $E\left(u^{\prime}\left(S_{1}^{1}\right)\left(S_{1}^{1}-1\right)\right)=1-E\left(1 / S_{1}^{1}\right)>0$.

In the context of neutral derivative pricing we consider the following extension:

Example 3.2 In the setup of Example 3.1 we consider a contingent claim whose payoff $H$ is independent of $S_{1}^{1}$ and satisfies $P(H=0)=1 / 2=P(H=1)$. Let $S_{0}^{2}$ denote the price of this option at time 0. By [17], Lemma 2.2 it remains optimal to buy one share of $S^{1}$ in the extended market if and only if

$$
E\left(\frac{1}{S_{1}^{1}}\left(\left(\psi^{1}-1\right)\left(S_{1}^{1}-1\right)+\psi^{2}\left(H-S_{0}^{2}\right)\right)\right) \leq 0
$$

holds for all $\left(\psi^{1}, \psi^{2}\right)$ with expected utility $E\left(\log \left(1+\psi^{1}\left(S_{1}^{1}-1\right)+\psi^{2}\left(H-S_{0}^{2}\right)\right)\right)>-\infty$. If $\psi^{2} \geq 0$, then only $-\left(1-\psi^{2} S_{0}^{2}\right)<\psi^{1} \leq 1-\psi^{2} S_{0}^{2}$ leads to finite expected utility. Similarly, we must have $-\left(1+\psi^{2}\left(1-S_{0}^{2}\right)\right)<\psi^{1} \leq 1+\psi^{2}\left(1-S_{0}^{2}\right)$ for $\psi^{2}<0$. Since the left-hand side of (3.1) equals

$$
\begin{aligned}
& E\left(1 / S_{1}^{1}\right)\left(\frac{1}{2}-S_{0}^{2}\right) \psi^{2}-\left(1-E\left(1 / S_{1}^{1}\right)\right)\left(1-\psi^{1}\right) \\
& \quad \leq \begin{cases}E\left(1 / S_{1}^{1}\right)\left(\frac{1}{2}-S_{0}^{2}\right) \psi^{2}-\left(1-E\left(1 / S_{1}^{1}\right)\right) \psi^{2} S_{0}^{2} & \text { if } \psi^{2} \geq 0 \\
E\left(1 / S_{1}^{1}\right)\left(\frac{1}{2}-S_{0}^{2}\right) \psi^{2}+\left(1-E\left(1 / S_{1}^{1}\right)\right) \psi^{2}\left(1-S_{0}^{2}\right) & \text { if } \psi^{2}<0\end{cases}
\end{aligned}
$$

one easily concludes that $(1,0)$ is an optimal strategy for $S=\left(S^{1}, S^{2}\right)$ if and only if

$$
S_{0}^{2} \in\left[\frac{1}{2} E\left(1 / S_{1}^{1}\right), 1-\frac{1}{2} E\left(1 / S_{1}^{1}\right)\right] .
$$

In view of Theorem 2.5, this interval of neutral prices coincides with

$$
\left[\inf _{Q \in \mathscr{Q}}\langle Q, H\rangle, \sup _{Q \in \mathscr{Q}}\langle Q, H\rangle\right],
$$

where $\mathscr{Q}$ denotes the set of neutral set functions for $S^{1}$. From the definition of neutral set functions it follows that $\frac{d Q^{r}}{d P}=1 / S_{1}^{1}$ and hence $\left\langle Q^{s}, 1\right\rangle=1-E\left(1 / S_{1}^{1}\right)$ for any $Q \in \mathscr{Q}$. The independence of $H$ and $S_{1}^{1}$ yields $E_{Q^{r}}(H)=\frac{1}{2} E\left(1 / S_{1}^{1}\right)$. Since the length of the interval of neutral prices equals the loss of measure, one easily concludes that $\left\langle Q^{s}, H\right\rangle$ assumes any value between 0 and $\left\langle Q^{s}, 1\right\rangle$. If there was no loss of measure, $S_{0}^{2}=\frac{1}{2}$ would be the unique neutral price of the claim $H$.

\subsection{Intermediate trades matter}

The utility-based valuation concept of [13] is closely connected to neutral pricing. In both approaches contingent claims are priced such that investment in these derivatives does not 
increase expected utility. The concepts differ in the precise meaning of investment. We allow for dynamic trading in all securities, whereas this is limited to the underlyings in [13]. One may expect that marginal utility-based prices in the sense of [13] can always be extended to neutral price processes. However, this does not hold in general, which may seem counterintuitive on first glance: No matter how a certain contingent claim is priced in the periods strictly between time 0 and maturity, dynamic investment in this claim will always be profitable even though it possesses a marginal utility-based initial price.

Example 3.3 We consider a two-period modification of Example 3.2. The claim $H$ expires at time $t=2$ but the stochastic outcome of the underlying is revealed already at $t=1$ (i.e. $S_{1}^{1}=S_{2}^{1}$ is as in Examples 3.1 and 3.2). Consequently, the filtration $\left(\mathscr{F}_{t}\right)_{t=0,1,2}$ is given by $\mathscr{F}_{0}=\{\varnothing, \Omega\}, \mathscr{F}_{1}:=\sigma\left(S_{1}^{1}\right), \mathscr{F}_{2}:=\sigma\left(S_{1}^{1}, H\right)$. Observe that the set of payoffs of admissible strategies in the underlying market and the set $\mathscr{Q}$ of neutral set functions remains the same as in Example 3.2. Nevertheless, we obtain now a unique neutral derivative price process for $H$, namely $S_{0}^{2}=S_{1}^{2}=1 / 2$ :

The existence follows from Theorem 2.5. Denote by $S^{2}$ an arbitrary neutral derivative price process. Denote by $\pi_{k}$ the price $S_{1}^{2}$ of the claim $H$ on the set $\left\{S_{1}^{1}=x_{k}\right\}$. By Theorem 2.5 , we have

$$
\pi_{k}=\frac{\left\langle Q, 1_{\left\{S_{1}^{1}=x_{k}\right\}} H\right\rangle}{\left\langle Q, 1_{\left\{S_{1}^{1}=x_{k}\right\}}\right\rangle}
$$

for some neutral set function $Q \in \mathscr{Q}$. Since the singular part of $Q$ vanishes on $\left\{S_{1}^{1}=x_{k}\right\}$, we have

$$
\pi_{k}=\frac{\left\langle Q^{r}, 1_{\left\{S_{1}^{1}=x_{k}\right\}} H\right\rangle}{\left\langle Q^{r}, 1_{\left\{S_{1}^{1}=x_{k}\right\}}\right\rangle}=\frac{E\left(\frac{1}{S_{1}^{1}} 1_{\left\{S_{1}^{1}=x_{k}\right\}} H\right)}{E\left(\frac{1}{S_{1}^{1}} 1_{\left\{S_{1}^{1}=x_{k}\right\}}\right)}=\frac{1}{2},
$$

i.e. $S_{1}^{2}=1 / 2$ is the unique neutral derivative price at time 1 . Absence of arbitrage or another application of Theorem 2.5 yields that $S_{0}^{2}=1 / 2$ as well.

Since intermediate trades of the claim are not intended in the approach of [13], Examples 3.2 and 3.3 lead to the same set of marginal utility-based derivative prices $S_{0}^{2}$. This set of prices equals the interval of initial neutral prices in Example 3.2 because the two valuation concepts coincide in one-period markets. Consequently, Example 3.3 shows that there are marginal utility-based prices $S_{0}^{2}$ which cannot be extended to some neutral price process $\left(S_{0}^{2}, S_{1}^{2}, S_{2}^{2}\right)$ with $S_{2}^{2}=H$. Moreover, there are some $Q \in \mathscr{Q}$ such that there exists no $Q$-martingale with terminal value $H$. In particular, the recursive construction in the proof of Theorem 2.5 does not necessarily lead to a martingale under the neutral set function $Q$ that was chosen in Step 1.

Recall, however, that these phenomena occur only if the dual minimizer $Q$ fails to be a probability measure. Otherwise, both the marginal utility-based derivative price and the neutral price process are unique. They are derived by (conditional) expectation under this neutral pricing measure $Q$ (cf. $[13,17]$ ). 


\subsection{Continuous-time neutral prices may not exist}

We turn now to the question whether Theorem 2.5 can be extended to continuous-time. Surprisingly, this is not the case as the following example shows. It starts with a market with one risky security satisfying NFLVR and having finite expected logarithmic utility. In this market we explicitly construct a bounded claim which does not allow for a neutral price process.

Example 3.4 Step 1: Denote by $X_{1}$ a random variable as in Example 3.1, i.e. assuming the values $\left(x_{k}\right)_{k \in \mathbb{N}}$ with probabilities $\left(p_{k}\right)_{k \in \mathbb{N}}$, where $x_{0}:=2, p_{0}:=5 / 6, x_{k}:=2^{-k}$, $p_{k}:=4^{-k} / 2$ for $k \geq 1$. Let $0=t_{0}<t_{1}<\ldots<t_{\infty}=1$. We construct a model in countable discrete time $t=t_{0}, t_{1}, \ldots, t_{\infty}$, which can be extended to the whole continuous time interval $[0,1]$ through the usual right-continuous extension. To this end, let $X_{0}:=1$ and

$$
X_{t_{2 k+2}}:=X_{t_{2 k+1}}:= \begin{cases}X_{1} & \text { if } X_{1} \in\left\{x_{0}, \ldots, x_{k}\right\} \\ s_{k} & \text { if } X_{1}<x_{k}\end{cases}
$$

for $k \in \mathbb{N}$, where

$$
s_{k}:=\frac{1}{2^{k}\left(2^{k-1}+3\right)} .
$$

In addition, denote by $\left(U_{k}\right)_{k \in \mathbb{N}}$ a sequence of independent binary random variables, also independent of $X_{1}$, with $P\left(U_{k}=0\right)=q_{k}=1-P\left(U_{k}=1\right)$, where

$$
q_{k}:=\frac{2^{-(k+1)}}{1+2^{-k}}
$$

The filtration $\left(\mathscr{F}_{t_{n}}\right)_{n \in \mathbb{N} \cup\{\infty\}}$ is chosen as

$$
\begin{aligned}
\mathscr{F}_{0} & :=\{\varnothing, \Omega\} \\
\mathscr{F}_{t_{2 k+1}} & :=\sigma\left(X_{t_{0}}, \ldots, X_{t_{2 k+1}}, U_{0}, \ldots, U_{k-1}\right), \\
\mathscr{F}_{t_{2 k+2}} & :=\sigma\left(X_{t_{0}}, \ldots, X_{t_{2 k+2}}, U_{0}, \ldots, U_{k}\right), \\
\mathscr{F}_{1} & :=\sigma\left(X_{t_{0}}, \ldots, X_{t_{\infty}}, U_{0}, U_{1}, \ldots\right) .
\end{aligned}
$$

Define $\kappa_{0}:=\inf \left\{k \in \mathbb{N}: U_{k}=0\right\}$ and observe that $\sigma:=t_{2 \kappa_{0}+2}$ is a stopping time. Similarly, let $\kappa_{1}:=\inf \left\{k \in \mathbb{N}: X_{t_{2 k+1}}=x_{k}\right\}$ and define the stopping time $\tau:=t_{2 \kappa_{1}+1}$. As risky asset we consider the stopped process $S^{1}:=X^{\sigma}$.

Step 2: We show that $S^{1}$ satisfies Condition NFLVR. To this end, observe that $\left(1 / X_{t_{n}}\right)_{n \in \mathbb{N}}$ is a martingale relative to $\left(\mathscr{F}_{t_{n}}\right)_{n \in \mathbb{N}}\left(\right.$ excluding $\left.t_{\infty}\right)$. Set

$$
Y_{k}= \begin{cases}\left(2^{k+1}+3\right) / 2 & \text { if } U_{k}=0, \\ 1 / 2 & \text { if } U_{k}=1\end{cases}
$$

for $k \in \mathbb{N}$, which implies that $E\left(Y_{k}\right)=1$. Moreover, define a uniformly integrable martingale $M$ via $M_{t_{1}}:=M_{0}:=1$,

$$
M_{t_{2 k+3}}:=M_{t_{2 k+2}}:=\prod_{i=0}^{k \wedge \kappa_{0}} Y_{i}
$$


for $k \in \mathbb{N}$ and $M_{1}:=\left(1+3 / 2^{\kappa_{0}+1}\right) 1_{\left\{\kappa_{0}<\infty\right\}}$. If we set

$$
Z:=\frac{M}{S^{1}}=\frac{M}{X^{\sigma}},
$$

then $\left(Z_{t_{n}}\right)_{n \in \mathbb{N}}$ is a positive martingale relative to $\left(\mathscr{F}_{t_{n}}\right)_{n \in \mathbb{N}}$ because $M$ and $X$ change only in even and odd periods, respectively. Moreover, it converges almost surely to $Z_{1}$. The independence of $X$ and $\sigma$ yields

$$
E\left(Z_{1}\right)=E\left(E\left(Z_{1} \mid \sigma\right)\right)=\sum_{k \in \mathbb{N}} P\left(\sigma=t_{2 k+2}\right) \frac{2^{k+1}+3}{2^{k+1}} E\left(\frac{1}{X_{t_{2 k+2}}}\right) .
$$

Since

$$
P\left(\sigma=t_{2 k+2}\right)=\prod_{i=0}^{k-1}\left(1-q_{i}\right) q_{k}=2^{-k-2}
$$

and $E\left(1 / X_{t_{2 k+2}}\right)=1$, it follows that $E\left(Z_{1}\right)=E\left(Z_{0}\right)$. Therefore $\left(Z_{t_{n}}\right)_{n \in \mathbb{N} \cup\{\infty\}}$ is a uniformly integrable martingale such that $Z S^{1}$ is a martingale. Consequently, $\frac{d Q}{d P}:=Z_{1}$ defines an equivalent martingale measure $Q$, which shows NFLVR.

Step 3: Suppose that the investor has logarithmic utility and initial endowment 1 . Since $1 / S^{1}$ is a local martingale, it follows that holding one share of $S^{1}$ is an optimal strategy in the market $S^{1}$ (cf. [11], Lemma 2.3).

Let us now introduce a European claim with payoff $H:=1_{\left\{\kappa_{0}=\infty\right\}}$. Suppose that there exists some neutral derivative price process $S^{2}$ for $H$. We will show in Step 4 that this leads to a contradiction. By absence of arbitrage $S^{2}$ must be [0,1]-valued and $(0,1]$-valued before $\sigma$ because $S_{1}^{2}=H$ may still end up positive with positive probability. From $\sigma$ on, $S^{2}$ obviously equals 0 unless $\sigma=1$.

Since $S^{1}$ is the wealth process of an optimal strategy, both $S^{2} / S^{1}$ and $-S^{2} / S^{1}$ are supermartingales by [1], Proposition 4.3. From $S_{t_{2 k+2}}^{1}=S_{t_{2 k+1}}^{1}$ it follows that

$$
E\left(S_{t_{2 k+2}}^{2}-S_{t_{2 k+1}}^{2} \mid \mathscr{F}_{t_{2 k+1}}\right)=0
$$

for any $k \in \mathbb{N}$.

Step 4: Define a process $V$ by

$$
\begin{aligned}
V_{0} & :=0, \\
V_{t_{2 k+1}} & := \begin{cases}1-\frac{24}{\left(1+2^{-k}\right)\left(1+3 / 2^{k-1}\right)} & \text { if } t_{2 k+1}<\sigma \wedge \tau, \\
1 & \text { otherwise, }\end{cases} \\
V_{t_{2 k+2}} & := \begin{cases}1-\frac{24}{\left(1+2^{-k-1}\right)\left(1+3 / 2^{k-1}\right)} & \text { if } t_{2 k+1}<\sigma \wedge \tau, \\
1 & \text { otherwise, }\end{cases} \\
V_{1} & :=1
\end{aligned}
$$

for $k \in \mathbb{N}$. Moreover, we denote by $\varphi=\left(\varphi^{1}, \varphi^{2}\right)$ a trading strategy for $\left(S^{1}, S^{2}\right)$ with $\varphi=0$ on $\rrbracket \sigma \wedge \tau, 1 \rrbracket$. On $\llbracket 0, \sigma \wedge \tau \rrbracket$ it is defined via

$$
\varphi_{t_{1}}:=(1,0)
$$




$$
\begin{aligned}
\varphi_{t_{2 k+1}} & :=\left(\frac{24 \cdot 2^{k}}{\left(1+2^{-k}\right)\left(1+2^{-k+2}\right)}, 0\right) \quad \text { for } k=1,2, \ldots, \\
\varphi_{t_{2 k+2}} & :=\left(0, \frac{-24}{\left(1+2^{-k}\right)\left(1+3 / 2^{k-1}\right) S_{t_{2 k+1}}^{2}}\right) \text { for } k=0,1,2, \ldots
\end{aligned}
$$

Using (3.2) one verifies by straightforward induction that $V$ is the wealth process for $\varphi$ corresponding to initial capital 0 . Since $V$ is bounded from below by -23 , the strategy $\varphi$ is an arbitrage, which implies that the maximal expected logarithmic utility in the extended market $\left(S^{1}, S^{2}\right)$ is infinite. Consequently, $S^{2}$ cannot be a neutral price process.

Remark 3.5 The value process of the arbitrage strategy in the previous example is a local $Q$-martingale for all $Q \in \mathscr{Q}$. But it is not a $Q$-supermartingale although it is bounded from below. The strategy can be interpreted as an investment in a sequence of nonnegative claims $Y^{n}$ with $\left\langle Q^{s}, Y^{n}\right\rangle=0$.

Let us have a look at how the situation changes if admissible strategies are by definition assumed to have nonnegative wealth $v+\psi \cdot S$ in the whole interval $[0, T]$. It is an interesting question whether neutral price processes generally exist in this slightly different context. If such a modified neutral price process exists in the previous example, it leads to arbitrage. Indeed, in any market without arbitrage opportunities, the two notions of admissibility result in the same optimal strategies because the more general admissible portfolios in this paper are assigned expected utility $-\infty$. Since we consider arbitrage as unacceptable for any reasonable valuation approach, we do not discuss the above question further.

\section{Neutral pricing for utility functions on $\mathbb{R}$}

In this section we consider utility functions which are finite on the whole real line, the prime example being exponential utility $u(x)=-e^{-x}$. The general setup is as in the first two paragraphs of Section 2. We assume that investors are identical utility maximizers with initial endowment $v \in \mathbb{R}$. Their preferences are modelled by a continuously differentiable, strictly concave utility function $u: \mathbb{R} \rightarrow \mathbb{R}$ which satisfies $\lim _{x \rightarrow-\infty} u^{\prime}(x)=\infty$ and $u^{\prime}(\infty)=\lim _{x \rightarrow \infty} u^{\prime}(x)=0$. For this type of utility functions the proper choice of the set of admissible trading strategies is a delicate issue. On the one hand one should allow for terminal wealths unbounded from below as utility is finite everywhere. On the other hand some stochastic credit line is needed in order to exclude e.g. favourable doubling strategies.

We work in the setup of [2], which extends and generalizes earlier work to the case of possibly non-locally bounded processes. The idea is to consider $W$-admissible strategies $\varphi \in L(S)$ in the sense that the process $\varphi \cdot S$ is bounded from below by $-c W$ for some $c \in \mathbb{R}_{+}$. Here, $W \geq 1$ denotes some $u$-compatible and $S$-suitable random variable. The results are independent of its particular choice. $u$-compatibility means that $E(u(-c W))>$ 
$-\infty$ for any $c \in \mathbb{R}_{+}$. $W$ is called $S$-suitable if for any $i=1, \ldots, m+n$ there exists some $H \in L\left(S^{i}\right)$ such that $\{H=0\}$ is evanescent and

$$
-W \leq H \cdot S^{i} \leq W
$$

Since the derivatives price process $\left(S^{m+1}, \ldots, S^{m+n}\right)$ is assumed to be bounded, it suffices to assume (4.1) for $i=1, \ldots, m$. In order to obtain reasonable results, we make two assumptions on the optimization problem in the underlyings' market (cf. [2]). Firstly, we suppose

$$
E\left(\Phi\left(\lambda \frac{d Q}{d P}\right)\right)<\infty
$$

for any $\lambda>0$ and any $Q \in \mathscr{Q}$. Here, $\Phi(y):=\sup _{x \in \mathbb{R}}(u(x)-x y)$ denotes the convex conjugate of $u$ and

$$
\mathscr{Q}:=\left\{Q \ll P: S^{1}, \ldots, S^{m} \text { are } Q-\sigma \text {-martingales and } E\left(\Phi\left(\frac{d Q}{d P}\right)\right)<\infty\right\}
$$

stands for the domain of a dual minimization problem. Moreover, the maximal utility which is achievable by trading in the underlyings should not be too large, namely

$$
\sup \left\{E\left(u\left(v+\varphi \cdot S_{T}\right)\right): \varphi W \text {-admissible with } \varphi^{m+1}=\ldots=\varphi^{m+n}=0\right\}<u(\infty) .
$$

The supremum in (4.4) may not be attained by any $W$-admissible strategy. But under the above assumptions we have

$$
\begin{aligned}
& \sup \left\{E\left(u\left(v+\varphi \cdot S_{T}\right)\right): \varphi W \text {-admissible with } \varphi^{m+1}=\ldots=\varphi^{m+n}=0\right\} \\
= & E\left(u\left(v+f^{*}\right)\right)
\end{aligned}
$$

for a unique random variable

$$
f^{*} \in K_{\Phi}:=\left\{f \in \bigcap_{Q \in \mathscr{Q}} L^{1}(Q): E_{Q}(f) \leq 0, \forall Q \in \mathscr{Q}\right\},
$$

which may attain the value $\infty$ with positive probability (cf. [2], Theorem 1). We call the measure $Q^{*} \in \mathscr{Q}$ with density

$$
\frac{d Q^{*}}{d P}:=\frac{u^{\prime}\left(v+f^{*}\right)}{E\left(u^{\prime}\left(v+f^{*}\right)\right)}
$$

neutral pricing measure. It solves some dual minimization problem and plays a similar role as the neutral set functions in Definition 2.3.

As in Section 2 neutral pricing is supposed to indicate that trading derivatives does not increase expected utility. Since the optimal utility in (4.4) may not be attained by $W$ admissible trading, we must modify Definition 2.2 slightly. 
Definition 4.1 We call a derivatives price process $\left(S^{m+1}, \ldots, S^{m+n}\right)$ neutral price process if

$$
\begin{aligned}
& \sup \left\{E\left(u\left(v+\varphi \cdot S_{T}\right)\right): \varphi W \text {-admissible }\right\} \\
& =\sup \left\{E\left(u\left(v+\varphi \cdot S_{T}\right)\right): \varphi W \text {-admissible with } \varphi^{m+1}=\ldots=\varphi^{m+n}=0\right\} .
\end{aligned}
$$

Theorem 4.2 The set of neutral price processes $\left(S^{m+1}, \ldots, S^{m+n}\right)$ coincides with the set of derivative price processes that are $Q^{*}$-martingales with terminal value $\left(H^{1}, \ldots, H^{n}\right)$. If they exist, neutral price processes are unique up to $Q^{*}$-indistinguishability or, equivalently, up to $P$-indistinguishability on the set $\{Z \neq 0\}$, where $Z$ denotes the density process of $Q^{*}$ relative to $P$.

Proof. Suppose that $\left(S^{m+1}, \ldots, S^{m+n}\right)$ is a neutral derivatives price process. Define $\widetilde{\mathscr{Q}}$ and $\widetilde{K}_{\Phi}$ as in (4.3) and (4.5) but corresponding to the large market, i.e. such that $S^{i}, i \leq m+n$ rather than only $S^{i}, i \leq m$ are required to be $Q$ - $\sigma$-martingales. Obviously, we have $\widetilde{\mathscr{Q}} \subset \mathscr{Q}$ and $K_{\Phi} \subset \widetilde{K}_{\Phi}$. From

$$
\sup \left\{E\left(u\left(v+\varphi \cdot S_{T}\right)\right): \varphi W \text {-admissible }\right\}=E\left(u\left(v+f^{*}\right)\right)
$$

and Theorem 1 in [2] it follows that $Q^{*} \in \widetilde{\mathscr{Q}}$. Therefore $S^{m+1}, \ldots, S^{m+n}$ are $Q^{*}$ - $\sigma$-martingales and hence $Q^{*}$-martingales because they are bounded. In particular, they are up to $Q^{*}$ indistinguishability unique.

In order to show the converse, let $S^{m+1}, \ldots, S^{m+n}$ be semimartingales which are at the same time bounded $Q^{*}$-martingales with terminal values $H^{1}, \ldots, H^{n}$. If we define $\widetilde{\mathscr{Q}}$ and $\widetilde{K}_{\Phi}$ as in the first part of the proof, we have $Q^{*} \in \widetilde{\mathscr{Q}}$ because $S^{m+1}, \ldots, S^{m+n}$ are $Q^{*}$-martingales. Estimation (11) in [2] yields that

$$
\sup \left\{E\left(u\left(v+\varphi \cdot S_{T}\right)\right): \varphi W \text {-admissible }\right\}<u(\infty) .
$$

Moreover, $Q^{*}$ solves the dual minimization problem in [2] both on $\mathscr{Q}$ and on the smaller set $\widetilde{\mathscr{Q}}$, which implies that the optimal value of the utility maximization problem in the small and in the large market coincide (cf. [2], Theorem 1). Put differently, Equation (4.6) holds.

If $\mathscr{Q}$ contains some measure which is equivalent to $P$, then $Q^{*} \sim P$ for the neutral pricing measure above (cf. Remark 2 in [2]). In this case, neutral price processes exist and they are unique up to $P$-indistinguishability. But even in the general case the non-uniqueness in Theorem 4.2 is of a different kind than the one in Theorem 2.5. In the latter case, it is due to non-uniqueness of the dual minimizer. Here, however, non-unique prices are caused by non-equivalence of $P$ and $Q^{*}$. Ambiguous option prices occur only on the set where the density process of $Q^{*}$ relative to $P$ vanishes. On this set you are infinitely rich at $T$, which implies that you do not care about additional profit. If the initial $\sigma$-field $\mathscr{F}_{0}$ is trivial, the initial prices $\left(S_{0}^{m+1}, \ldots, S_{0}^{m+n}\right)$ are unique. 
In arbitrage-free discrete-time markets (i.e. with time domain $\{0,1, \ldots, T\}$ ) satisfying (4.2) and (4.4), unique neutral derivative prices exist. Indeed, by the fundamental theorem of asset pricing (cf. [4]) there exists some martingale measure $Q \sim P$ with bounded density. Since $\Phi$ is continuous with $\lim _{x \rightarrow 0} \Phi(x)=u(\infty)$, this implies $Q \in \mathscr{Q}$ and hence $Q^{*} \sim P$ if $u(\infty)<\infty$. If $u(\infty)=\infty$, the equivalence $Q^{*} \sim P$ holds by Remark 2 in [2].

The following example shows that neutral price processes may fail to exist in continuous time. The point is that no version of the $Q^{*}$-martingale in Theorem 4.2 is a $P$ semimartingale.

Example 4.3 We consider a single underlying $S^{1}$ with time horizon $T=1$. The stock $S^{1}$ grows linearly according to $S_{t}^{1}=1+t$ up to a random jump time $\tau$ where it has a jump of size $\Delta S_{\tau}^{1}=-(1-\tau)$ and stops, i.e. remains constant afterwards. We start by modelling $S^{1}$ under probability measure $Q$. Relative to $Q$ the jump intensity at time $t$ is supposed to be $\lambda_{t}:=1 /(1-t)$. Since $\int_{0}^{1} \lambda_{t} d t=\infty$, we have that the stock jumps $Q$-almost surely. One easily verifies that $S^{1}$ is a $Q$-martingale. Moreover, we have

$$
Q(\tau>t)=\exp \left(-\int_{0}^{t} \lambda_{s} d s\right)=1-t
$$

for $0 \leq t \leq 1$ and, more generally,

$$
Q(\tau>t \mid \tau>s)=\frac{1-t}{1-s}
$$

for $0 \leq s<t \leq 1$.

We consider an investor with initial endowment $v=0$ and exponential utility function $u(x)=-e^{-x}$. We choose $W=1$ for the definition of admissibility. Define a deterministic trading strategy

$$
\varphi_{t}:=\frac{1}{2(1-t)}
$$

which leads to the wealth process

$$
V_{t}:=\varphi \cdot S_{t}^{1}= \begin{cases}-\frac{1}{2} \log (1-t) & \text { for } t<\tau \\ -\frac{1}{2} \log (1-\tau)-\frac{1}{2} & \text { for } t \geq \tau\end{cases}
$$

In particular, we have

$$
E_{Q}\left(\exp \left(V_{T}\right)\right)=\int_{0}^{1} \exp \left(-\frac{1}{2} \log (1-t)-\frac{1}{2}\right) d t=\frac{2}{\sqrt{e}} .
$$

Finally, we define the objective probability measure $P$ by its density

$$
\frac{d P}{d Q}:=\frac{\sqrt{e}}{4} \exp \left(V_{T}\right)
$$

Note that $E_{Q}\left(\frac{d P}{d Q}\right)=1 / 2<1$, which means that $P$ is not a probability measure yet. The missing mass $1 / 2$ is assigned to the path where no jump happens, which means that $P$ becomes a true probability measure with $Q \ll P$. Note that

$$
\frac{d Q}{d P}=\frac{4}{\sqrt{e}} u^{\prime}\left(V_{T}\right)
$$


holds also on the set $\{\tau<1\}^{C}$ and hence $P$-almost surely. Dominated convergence yields $V_{t} \rightarrow V_{T}$ in $L^{1}(Q)$ for $t \rightarrow T$, which implies $E_{Q}\left(V_{T}\right)=0$. For any $W$-admissible $\psi$ we have

$$
\begin{aligned}
E_{P}\left(u\left(\psi \cdot S_{T}^{1}\right)\right) & \leq E_{P}\left(u\left(V_{T}\right)\right)+E_{P}\left(u^{\prime}\left(V_{T}\right)\left(\psi \cdot S_{T}^{1}-V_{T}\right)\right) \\
& =E_{P}\left(u\left(V_{T}\right)\right)+\frac{\sqrt{e}}{4}\left(E_{Q}\left(\psi \cdot S_{T}^{1}\right)-E_{Q}\left(V_{T}\right)\right) \\
& \leq E_{P}\left(u\left(V_{T}\right)\right)<u(\infty) .
\end{aligned}
$$

Consequently, (4.4) holds. Condition (4.2) is generally satisfied for exponential utility by [2], Section 2.2. Since $V$ is bounded from below and $V_{t} \in K_{\Phi}$ for any $t<T$, Fatou's lemma yields $V_{T} \in K_{\Phi}$. By (4.8) this implies that $f^{*}:=V_{T}$ is the optimal payoff of the utility maximization problem. (4.7) yields that $Q$ is the neutral pricing measure for this market model.

Consider now a contingent claim with payoff

$$
H:= \begin{cases}1 & \text { for } \tau \in \bigcup_{k=1,3,5, \ldots}\left(t_{k-1}, t_{k}\right] \\ 0 & \text { for } \tau \in \bigcup_{k=2,4,6, \ldots}\left(t_{k-1}, t_{k}\right] \cup\{\tau<1\}^{C}\end{cases}
$$

where $t_{k}:=1-3^{-k}$. Suppose that $S^{2}$ is a neutral price process for $H$ and hence a $Q$ martingale by Theorem 4.2. If $k$ is odd, we have

$$
\begin{aligned}
S_{t_{k-1}}^{2} & =E_{Q}\left(H \mid \mathscr{F}_{t_{k-1}}\right) \\
& =Q\left(H=1 \mid \tau>t_{k-1}\right) \\
& \geq Q\left(\tau \leq t_{k} \mid \tau>t_{k-1}\right) \\
& =1-\frac{3^{-k}}{3^{-k+1}} \\
& =\frac{2}{3}
\end{aligned}
$$

on the set $\left\{\tau>t_{k-1}\right\}$. Similarly, one concludes that $S_{t_{k-1}}^{2} \leq 1 / 3$ on the set $\left\{\tau>t_{k-1}\right\}$ if $k$ is even. Consequently, $S^{2}$ oscillates infinitely often between $1 / 3$ and $2 / 3$ on the set $\{\tau<1\}^{C}$, which has positive probability under $P$. On this set $S^{2}$ cannot have a left-hand limit in $t=1$. This in turn implies that it cannot be a $P$-semimartingale in contradiction to the definition of a derivative price process.

A close examination of the above example reveals that it allows for free lunch with vanishing risk under $P$. However, by slight modification one can turn it into a market satisfying NFLVR. One could e.g. modify the jump distribution by letting

$$
\Delta S_{t}^{1}:= \begin{cases}-(1-t) & \text { with jump intensity } \frac{1}{2(1-t)} \\ -1 & \text { with jump intensity } \frac{1}{2},\end{cases}
$$

under $Q$, which removes free lunches also under $P$. One may then proceed similarly as above with $\varphi_{t}:=\frac{1}{4(1-t)}$ and $t_{k}:=1-9^{-k}$. We leave the somewhat tedious technical details to the reader. 
Remark 4.4 Let us abandon the assumption that the payoffs $H^{i}$ are bounded and assume instead that any $H^{i}$ can be sub- and superhedged by a strategy in the underlyings. More precisely, we suppose

$$
k+\left(\varphi^{1}, \ldots, \varphi^{m}\right) \cdot\left(S^{1}, \ldots, S^{m}\right)_{T} \leq H^{i} \leq l+\left(\psi^{1}, \ldots, \psi^{m}\right) \cdot\left(S^{1}, \ldots, S^{m}\right)_{T}
$$

for some $k, l \in \mathbb{R}$ and some $\left(S^{1}, \ldots, S^{m}\right)$-integrable $\left(\varphi^{1}, \ldots, \varphi^{m}\right),\left(\psi^{1}, \ldots, \psi^{m}\right)$ such that $\left(\varphi^{1}, \ldots, \varphi^{m}\right) \cdot\left(S^{1}, \ldots, S^{m}\right)$ and $\left(\psi^{1}, \ldots, \psi^{m}\right) \cdot\left(S^{1}, \ldots, S^{m}\right)$ are true $Q^{*}$-martingales. Accordingly, we only suppose

$$
k+\left(\varphi^{1}, \ldots, \varphi^{m}\right) \cdot\left(S^{1}, \ldots, S^{m}\right) \leq S^{i} \leq l+\left(\psi^{1}, \ldots, \psi^{m}\right) \cdot\left(S^{1}, \ldots, S^{m}\right)
$$

instead of boundedness in the definition of a derivatives price process (cf. the second paragraph of Section 2). Then Theorem 4.2 continues to hold. This follows by combining arguments from the proofs of Corollary 3.5 in [17] and Theorem 4.2 above. From (4.10) one can conclude that $W$ remains suitable in the enlarged market.

Condition (4.10) for $k, l,\left(\varphi^{1}, \ldots, \varphi^{m}\right),\left(\psi^{1}, \ldots, \psi^{m}\right)$ satisfying (4.9) is a kind of reasonability condition for derivative price processes which appears similarly in [17]. Note that the implication $(4.9) \Rightarrow(4.10)$ does not follow from usual no-arbitrage arguments.

\section{Acknowledgements}

We are grateful to an anonymous referee of [17] for raising the question of existence and uniqueness addressed here. Moreover, we thank Walter Schachermayer and Mihai Sîrbu for valuable discussions and comments and two anonymous referees for helpful suggestions.

\section{References}

[1] D. Becherer. The numeraire portfolio for unbounded semimartingales. Finance \& Stochastics, 5:327-341, 2001.

[2] S. Biagini and M. Frittelli. Utility maximization in incomplete markets for unbounded processes. Finance \& Stochastics, 9:493-517, 2005.

[3] J. Cvitanić, W. Schachermayer, and H. Wang. Utility maximization in incomplete markets with random endowment. Finance \& Stochastics, 5:259-272, 2001.

[4] R. Dalang, A. Morton, and W. Willinger. Equivalent martingale measures and noarbitrage in stochastic security market models. Stochastics and Stochastics Reports, 29:185-202, 1990.

[5] M. Davis. Option pricing in incomplete markets. In M. Dempster and S. Pliska, editors, Mathematics of Derivative Securities, pages 216-226. Cambridge University Press, Cambridge, 1997. 
[6] M. Davis, V. Panas, and T. Zariphopoulou. European option pricing with transaction costs. SIAM Journal on Control and Optimization, 31:470-493, 1993.

[7] F. Delbaen and W. Schachermayer. A general version of the fundamental theorem of asset pricing. Mathematische Annalen, 300:463-520, 1994.

[8] N. Dunford and J. Schwartz. Linear Operators. Part I: General Theory. Wiley, New York, 1958.

[9] L. Foldes. Valuation and martingale properties of shadow prices: An exposition. Journal of Economic Dynamics \& Control, 24:1641-1701, 2000.

[10] M. Frittelli. The minimal entropy martingale measure and the valuation problem in incomplete markets. Technical Report 20, Università degli Studi di Milano, 1996.

[11] T. Goll and J. Kallsen. Optimal portfolios for logarithmic utility. Stochastic Processes and their Applications, 89:31-48, 2000.

[12] S. Hodges and A. Neuberger. Optimal replication of contingent claims under transaction costs. Review of Futures Markets, 8:222-239, 1989.

[13] J. Hugonnier, D. Kramkov, and W. Schachermayer. On utility-based pricing of contingent claims in incomplete markets. Mathematical Finance, 15:203-212, 2005.

[14] J. Jacod and A. Shiryaev. Limit Theorems for Stochastic Processes. Springer, Berlin, second edition, 2003.

[15] J. Kallsen. Semimartingale Modelling in Finance. Dissertation Universität Freiburg i. Br., 1998.

[16] J. Kallsen. Utility-based derivative pricing in incomplete markets. In H. Geman, D. Madan, S. Pliska, and T. Vorst, editors, Mathematical Finance - Bachelier Congress 2000, pages 313-338, Berlin, 2002. Springer.

[17] J. Kallsen and C. Kühn. Pricing derivatives of American and game type in incomplete markets. Finance \& Stochastics, 8:261-284, 2004.

[18] I. Karatzas and S. Kou. On the pricing of contingent claims under constraints. The Annals of Applied Probability, 6:321-369, 1996.

[19] D. Kramkov and W. Schachermayer. The asymptotic elasticity of utility functions and optimal investment in incomplete markets. The Annals of Applied Probability, 9:904950, 1999. 